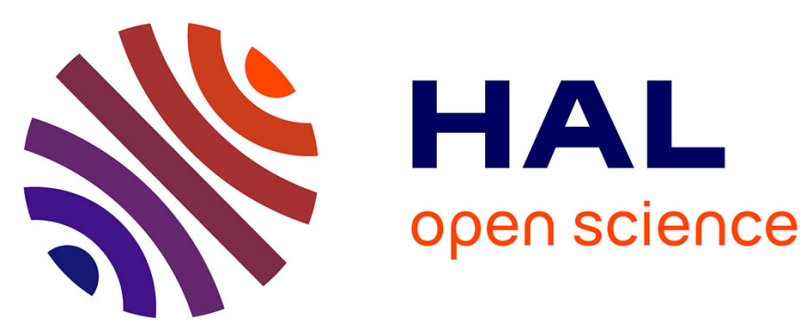

\title{
Experimental investigation and performance analysis of Archimedes screw generator
}

\author{
Guilhem Dellinger, Abdelali Terfous, Pierre-André Garambois, Abdallah \\ Ghenaim
}

\section{- To cite this version:}

Guilhem Dellinger, Abdelali Terfous, Pierre-André Garambois, Abdallah Ghenaim. Experimental investigation and performance analysis of Archimedes screw generator. Journal of Hydraulic Research, 2016, 54 (2), pp.197-209. 10.1080/00221686.2015.1136706 . hal-02350134

\section{HAL Id: hal-02350134 \\ https://hal.science/hal-02350134}

Submitted on 5 Nov 2019

HAL is a multi-disciplinary open access archive for the deposit and dissemination of scientific research documents, whether they are published or not. The documents may come from teaching and research institutions in France or abroad, or from public or private research centers.
L'archive ouverte pluridisciplinaire HAL, est destinée au dépôt et à la diffusion de documents scientifiques de niveau recherche, publiés ou non, émanant des établissements d'enseignement et de recherche français ou étrangers, des laboratoires publics ou privés. 
To appear in the Journal of Hydraulic Research

Vol. 00, No. 00, Month 20XX, 1-19

Research Paper

\title{
Experimental investigation and performance analysis of Archimedes Screw Generator
}

GUILHEM DELLINGER, PhD Student, Laboratoire des sciences de l'ingénieur, de l'informatique et de l'imagerie (ICUBE), Institut National des Sciences Appliquées (INSA) de Strasbourg, Strasbourg, France Email: guilhem.dellinger@insa-strasbourg.fr (author for correspondence)

ABDELALI TERFOUS (IAHR Member), Associate Professor, Laboratoire des sciences de l'ingénieur, de l'informatique et de l'imagerie (ICUBE), Institut National des Sciences Appliquées (INSA) de Strasbourg, Strasbourg, France

Email: abdelali.terfous@insa-strasbourg.fr

PIERRE-ANDRE GARAMBOIS, Assistant Professor, Laboratoire des sciences de l'ingénieur, de l'informatique et de l'imagerie (ICUBE), Institut National des Sciences Appliquées (INSA) de Strasbourg, Strasbourg, France

Email: pierre-andre.garambois@insa-strasbourg.fr

ABDELLAH GHENAIM, Professor, Laboratoire des sciences de l'ingénieur, de l'informatique et de l'imagerie (ICUBE), Institut National des Sciences Appliquées (INSA) de Strasbourg, France

Email: abdellah.ghenaim@insa-strasbourg.fr

\begin{abstract}
Generation of renewable energy with Archimedes Screw Generators (ASG) transforming potential energy of a fluid flow into mechanical energy is a growing technology convenient for low-head hydraulic sites. This paper presents an improved theoretical model linking screw performances to screw geometry and flow conditions. This model takes into account leakages, friction losses and variable fill levels. The modeled values of torques and efficiencies are in fairly good agreement with experimental results obtained for a laboratory-scale screw. The downstream screw immersion is shown to impact ASG efficiency and an optimal level is determined. It is also found that fluid friction on screw is not negligible and a friction coefficient is calibrated experimentally. Interestingly, it is found that a single value is suitable for modeling performances under various flow conditions. Leakage phenomenon in under filling flow conditions and friction forces in complex turbulent flows could be further studied.
\end{abstract}

Keywords: Archimedes screw generator; Experimental facilities; Hydraulic models; Hydraulics of renewable energy systems; Laboratory studies

\section{Introduction}

Finding new, safe, renewable energy is becoming more and more of a priority with global warming. One solution that is gaining popularity is the micro central hydraulics. Today, the microhydroelectricity has a large unexploited potential in Europe thanks to thousands of unused old mills or weirs. Among the many types of turbines, the Archimedes Screw Generator (ASG) is a developing way enabling the exploitation of these hydro sites. Known for more than 2000 years, the operating principle of the Archimedean screw was used as a pumping system to raise large amounts of water for low head sites. It is still used today for pumping wastewater in treatment plants. Its use as turbine for energy production was introduced for the first time by Radlik (1997). 
The first ASG was installed in 1997 on the Eger river in Aufhausen (Germany) and produces 4 $\mathrm{kW}$. There are now more than 180 hydropower plants of this type across Europe and more than 400 worldwide (Lashofer, Hawle, \& Pelikan, 2012), the most powerful ASG is in the Albert Canal (Belgium) and generates $400 \mathrm{~kW}$. This technology is still in its infancy and is a growing field.

According to the study by Williamson, Stark, and Booker (2014), the hydrodynamic screw has a high potential at low head and low flow sites. The maximum head and flow are respectively about $10 \mathrm{~m}$ and $10 \mathrm{~m}^{3} \mathrm{~s}^{-1}$. Among the main advantages of the ASG, high efficiencies can be maintained despite fluctuations of inflow discharge. According to Brada (1999), the flow rate can vary about $\pm 20 \%$ around the nominal flow without impacting efficiency. A recent study on 70 ASGs in Europe shows that the average in situ efficiency is about $69 \%$ with a maximum of $75 \%$ (Lashofer et al., 2012). An important point is its robustness; sediments and small debris can pass through an ASG without damaging it. The ASG is also usually assumed as fish friendly. Indeed, studies done on the river Dart have shown that with bumpers fitted on the leading edges, almost all kinds of fish can pass through the ASG without injuries (Kiebel, 2007, 2008; Kiebel \& Coe, 2011). However, the effects of ASGs on fish are still under discussion and in particular for small screw systems with high rotational speeds.

For design and optimization purposes, experimental and theoretical studies tried to link the ASG efficiency to its geometrical parameters and flow features. The experimental research conducted by Brada (1999) have shown the impact of speed and inflow water levels on ASG efficiency; however the downstream water level is not accounted despite its importance as shown in the present study. Lashofer, Hawle, and Pelikan (2013) tested experimentally the ASG performances for a large range of screw geometries. The impact of different geometrical parameters on screw efficiency was observed and it was concluded that the ASG design can still be improved. Laboratory tests were also performed on ASG with rotatory trough in order to eliminate leakage. Lower performances were found compared to an ASG with fixed trough, suggesting this diminution of performances is due to the friction between fluid and trough. About maximal ASG efficiencies in laboratory, Brada (1999) found results close to 80\%; Lubitz, Lyons, and Simmons (2014) and Lashofer et al. (2013) had results ranging between $80 \%$ and $90 \%$, whereas field efficiencies are on the order of $75 \%$ on average. This could be attributable to the fact that rotation speeds are generally not optimal for a given discharge. Moreover, river discharge is fluctuating in time, requiring and adaptive ASG speed to optimize performances.

Regarding geometry optimization, Rorres (2000) determined numerically the screw geometry maximizing the amount of water turbined in one turn of screw. Although these works have been done for Archimidean screw used as pump, these results are commonly used for ASG designing. Nuernbergk and Rorres (2012) developed an analytical model based on semi-empirical equations for the water inflow. It helps determine optimal values for inflow conditions given a screw geometry. This model can be useful to determine possible implantation sites. Assuming that the screw turns because of the water pressure (supposed hydrostatic) on the blades, Müller and Senior (2009) proposed a simplified theoretical model. The leakages are taken into account using the empirical equation given by Nagel and Radlik (1988). This equation was determined for an Archimedean screw used as a pump and operating in full condition. Their study shows that the screw efficiency is independent of the rotational speed. However, their model simplifies the screw geometry and does not take into account various other losses. Consequently, it is hard to use it for predicting a realistic ASG efficiency. Nuernbergk (2012) derived a model that takes into account leakage losses and the friction losses due to the viscosity of the fluid. The author considered a real screw geometry and an accurate leakage model that is not only based on empirical results. However, the variations of the water fill level in the screw are not taken into account. Hence, it is hard to determine the screw performances for different flow rates at fixed rotational speed or vice-versa. Recently Lubitz et al. (2014) proposed a model that takes into account the water fill level and the real screw geometry but friction losses are not included. Thus, by comparing their numerical and experimental results, the authors found a theoretical efficiency much higher than the experimental efficiency. Among the 
few references dealing with ASG, lots of questions remain especially for design purposes. There is still no comprehensive theoretical model on the Archimedean screw used as turbine. Today, the ASG designing is hence mainly based on empirical results.

This paper presents an improved theoretical model that takes into account the influence of leakage as proposed by Nuernbergk (2012), variable fill levels as highlighted by Lubitz et al. (2014), but also friction losses. In what follows, the hypothesis and calculations leading to the model are detailled. Then, experimental values measured on a laboratoratory scale screw are used for validating the efficiencies and torques determined with our model. Moreover, experimentations provide insights into the influence of various hydraulic and geometric parameters on the ASG performances. Finally, the fairly good capacities of the new model proposed for predicting the ASG performances and for optimizing its design are discussed.

The paper is structured as follows. The operating principle and notations are introduced in section 2. In section 3, the theoretical model is established for variable fill levels. The experimental device that allows modification of flow conditions and geometrical parameters is presented in section 4 . Results and discussions are exposed in section 5.

\section{Definition and operating principle}

An ASG consists in a screw rotating in an open and fix trough. The potential energy of the fluid flowing through the plant is transformed in mechanical energy thanks to the rotation of the screw. This mechanical energy is then transformed into electricity using a generator. The power provided by the ASG is given by

$$
P_{A S G}=\rho g Q H \eta_{A S G}
$$

where $P_{A S G}$ is the power, $\rho$ is the density of water, $Q$ is the flowrate, $g$ is the acceleration of gravity, $H$ is the geodetic head and $\eta_{A S G}$ is the efficiency of the whole system. The ASG efficiency is directly linked to thegenerator efficiency and to the different losses present in the plant. These main head losses, which result in power losses, are due to the leakages and frictional forces induced by the fluid viscosity. Minimizing these losses is thus important in order to obtain optimal efficiency.

The main features of an ASG are the head $H$, the total flowrate flowing through the plant $Q$, the rotational speed of the screw $n$ and the ASG efficiency $\eta_{A S G}$. The geometrical parameters of the screw shown in Fig. 1 are the outer radius $R_{a}$, the inner radius $R_{i}$, the pitch of the screw $S$, the total length $L$, the threaded length $L_{b}$, the number of blades $N$ and the screw inclination $\beta$. Like Rorres (2000), we define the volume of water trapped between two successive blades. This volume is named "bucket" and is equal to $V_{B}$. In the following analysis, all buckets present in a screw are assumed to be similar. Then, we define the optimal filling point of the screw which is reached when the filling level in a bucket is at the limit of overflowing in the next lower bucket. Figure 2 shows a screw at the optimal filling point with the different buckets.

By neglecting the various flow leakages, the flowrate flowing through the ASG is equal to the volume of water evacuated in one turn of screw multiplied by its rotational speed. Hence, we define the nominal flow $Q_{\text {nom }}$ by

$$
Q_{\text {nom }}=N V_{B} \frac{n}{60}
$$

where $n$ is the rotational speed of the screw. The nominal rotational speed $n_{n o m}$ is thus equal to

$$
n_{n o m}=\frac{Q_{n o m} 60}{N V_{B}}
$$




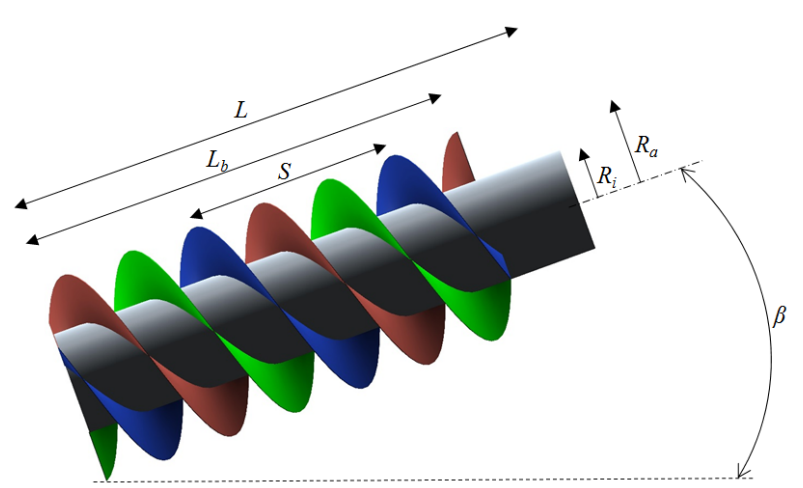

Figure 1 Geometrical parameters of the screw

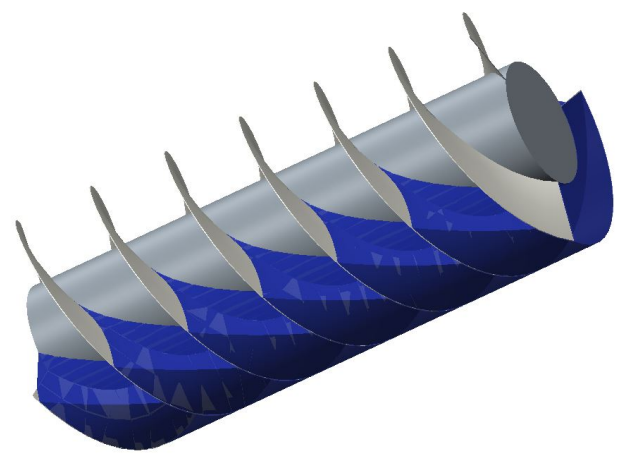

Figure 2 Archimedean screw at optimal filling point with the different buckets

\section{Theoretical model}

In order to design an ASG, it is important to be able to predict the axial torque provided by the screw and its efficiency - function of the flow features and geometrical parameters. As stated above, the model is established to explain how the different equations are extended to variable fill levels. The first part in the development of the theoretical model deals with the computation of the torque and the second one with the calculation of the different losses.

\subsection{Axial torque computation}

The axial torque delivered by the screw is due to the pressure, supposed hydrostatic, exerted by the water on each blade of the screw. A hydrostatic pressure is exerted on one blade by its upper and lower bucket. The pressure due to the upper bucket will lead to a motor torque on the screw axis. Conversely, the pressure due to the lower bucket will create a braking torque. Both torques are added to obtain the resulting torque delivered by one blade adjacent to two successive buckets within the screw.

We define the system $(\mathrm{O}, x, y, z)$ represented in Fig. 3. The lines (1), (2) and (3) represent respectively the water level for a screw in underfilling, at the optimal filling point and in overfilling. Knowing that the blades of the screw are helicoidal, for all point $M$ belonging to a blade surface with the change of variable $\varphi=2 \pi z / S$, we have

$$
\begin{gathered}
x_{M}=r_{M} \cos \left(\varphi_{M}\right) \\
y_{M}=r_{M} \sin \left(\varphi_{M}\right) \\
z_{M}=\frac{S}{2 \pi} \varphi_{M}
\end{gathered}
$$

where $r \in\left[R_{i}, R_{a}\right]$. Now, let us define the elementary force $\mathrm{d} f$ due to the hydrostatic pressure on a elementary surface $\mathrm{d} A$. An example of this elementary surface $\mathrm{d} A$ for a given point $M$ is represented in Fig. 3. We know that the direction of this force is orthogonal to the blade surface. Hence, $\mathrm{d} f=P_{\text {stat }} \mathrm{d} A$ where $P_{\text {stat }}$ is the hydrostatic pressure that is function of the water column above the elementary surface $\mathrm{d} A$. We use $\mathrm{d} A=\mathrm{d} b \mathrm{~d} r$ where $\mathrm{d} b$ is the elementary curvilinear length and $d r$ is the elementary radial length. For a parametric curve we have

$$
\mathrm{d} b=\sqrt{\left(\frac{\mathrm{d} x}{\mathrm{~d} \varphi}\right)^{2}+\left(\frac{\mathrm{d} y}{\mathrm{~d} \varphi}\right)^{2}+\left(\frac{\mathrm{d} z}{\mathrm{~d} \varphi}\right)^{2}}
$$




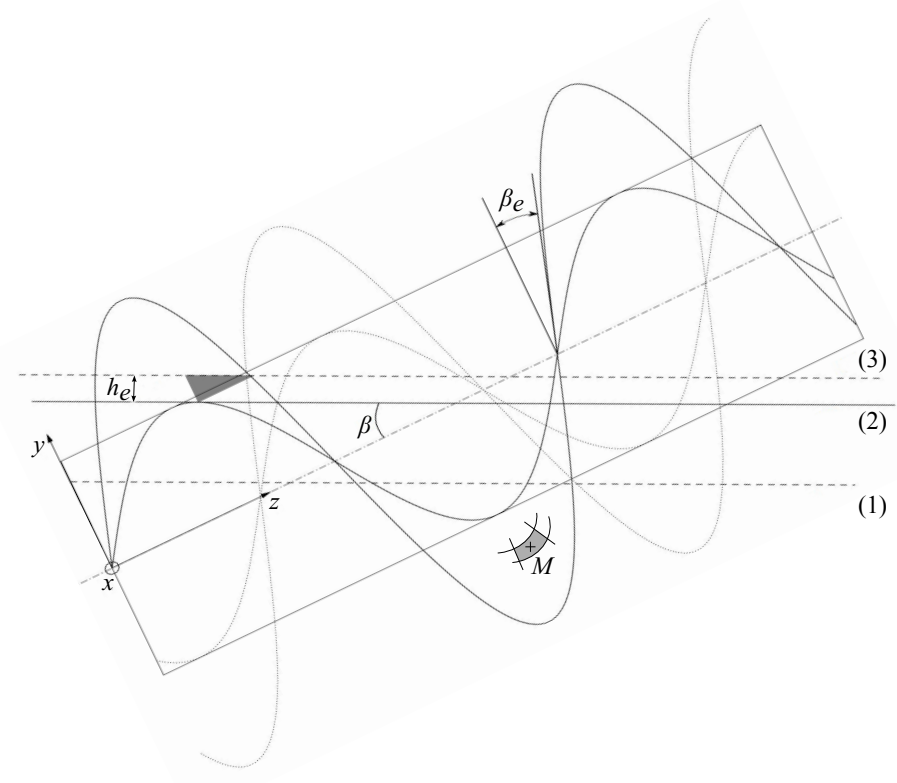

Figure 3 Archimedean screw profile in the system $(\mathrm{O}, x, y, z)$

Hence

$$
\mathrm{d} A=\mathrm{d} r \mathrm{~d} \varphi r \sqrt{1+\left(\frac{S}{2 \pi r}\right)^{2}}
$$

The hydrostatic pressure $P_{\text {stat }}$ for any point $M$ on the wetted surface of the blade is equal to

$$
P_{\text {stat }}(M)=\rho g H(M)=\rho g\left(y_{M}-h\left(z_{M}\right)\right)
$$

where $H(M)$ is the height of the water column above the point $M$ and $h\left(z_{M}\right)$ is the water level which is function of the screw filling. The elementary torque $\mathrm{d} C$ due to the force $\mathrm{d} f$ at $M$ is equal to

$$
\mathrm{d} C=\mathrm{d} f r \sin \left(\beta_{e}\right)
$$

where $\beta_{e}=\operatorname{cotan}(S / 2 \pi r)$ is the angle between the blade and the screw axis. This angle is represented in Fig. 3. The total torque is obtained by integrating the equation (7) on the upper and lower wetted surfaces of a blade. Finally, the torque delivered by one blade is equal to

$$
\begin{aligned}
C_{\text {th,blade }}= & \iint_{\text {surf,sup }} \rho g\left(y_{M}-h\left(z_{M}\right)\right) r \sin \left(\operatorname{cotan}\left(\frac{S}{2 \pi r}\right)\right) \mathrm{d} A \\
& -\iint_{\text {surf }, \text { inf }} \rho g\left(y_{M}-h\left(z_{M}\right)\right) r \sin \left(\operatorname{cotan}\left(\frac{S}{2 \pi r}\right)\right) \mathrm{d} A
\end{aligned}
$$

The torque provided by all the threaded length of the screw is

$$
C_{t h, \text { screw }}=C_{\text {th,blade }} \frac{L_{b} N}{S}
$$


It may be noted that the theoretical power output of the screw could be determined by

$$
P_{t h, s c r e w}=C_{t h, \text { screw }} \omega
$$

where $\omega$ is the rotational speed of the screw.

\subsection{Efficiency computation}

\section{Losses identification and efficiency}

Screw efficiency is directly linked to the different power losses that are non linear functions with respect to flow parameters. The different losses in an ASG have a direct influence on the screw efficiency and the efficiency is a non linear function of inflow discharge. As exposed by Nuernbergk and Rorres (2012), the power losses are due to leakage between the trough and the blades $P_{l}$, to leakage by over-filling $P_{\text {over }}$, to the friction between the water and the blades $P_{\text {blade }}$, to the friction between the water and the trough $P_{\text {trough }}$ and to the friction between the water and the screw core $P_{\text {core }}$. These losses are calculated by Nuernbergk (2012) for an ASG operating at an optimal filling point. Thus, if the flow rate varies, the rotational speed of the screw will change consequently as described by equation (3) and conversely. The ASG efficiency is thus given by

$$
\eta=1-\frac{\sum P_{\text {losses }}}{P_{\text {hydraulic }}}=1-\frac{P_{l}+P_{\text {over }}+P_{\text {blade }}+P_{\text {trough }}+P_{\text {core }}}{\rho g Q H}
$$

The total flowrate $Q$ is the sum of the nominal flow defined in equation (3) plus all the leakage flows. Then, we have

$$
Q=Q_{\text {nom }}+Q_{l}+Q_{\text {over }}
$$

where $Q_{l}$ is the leakage between the trough and the blades and $Q_{\text {over }}$ is the leakage by over-filling.

We propose here to use the main method of losses calculation and to extend it to variable screw filling. It will then be possible to determine the evolution of efficiency for a fixed flow rate and variable rotational speeds; and vice et versa.

\section{Leakage between trough and blades}

To determine the leakage gap, Muysken (1932) assumed that the leakage flow between two successive blades is similar to emptying a hydraulic tank. The author deduced the flow velocity, and knowing the gap between the blades and the trough, the leakage flowrate was calculated. This leakage is eventually determined by the following equation

$$
Q_{l}=\mu_{l} s_{s p} R_{a}\left(1+\frac{s_{s p}}{2 R_{a}}\right) \sqrt{1+\left(\frac{S}{2 \pi R_{a}}\right)^{2}}\left(\frac{2}{3} \alpha_{1}+\alpha_{2}+\frac{2}{3} \alpha_{3}\right) \sqrt{2 g \delta h}
$$

where $Q_{l}$ is the leakage flowrate and $s_{s p}$ is the gap between the blades and the trough. The constant $\mu_{l}$ is the contraction coefficient of discharge ranging between 0.65 and 1 . Here $\mu_{l}=1$ is chosen, i.e. for maximum gap leakage. The term $\delta h=S \sin (\beta) / N$ is the height difference between the water level of two successive buckets. The angles $\alpha_{1}, \alpha_{2}$ and $\alpha_{3}$ are drawn in Fig. 4 and are numerically determined. The angles $\alpha_{1}$ and $\alpha_{3}$ correspond to the blade area that is only under the pressure of an upper bucket. The pressures of an upper and lower bucket are exerted on an area corresponding 


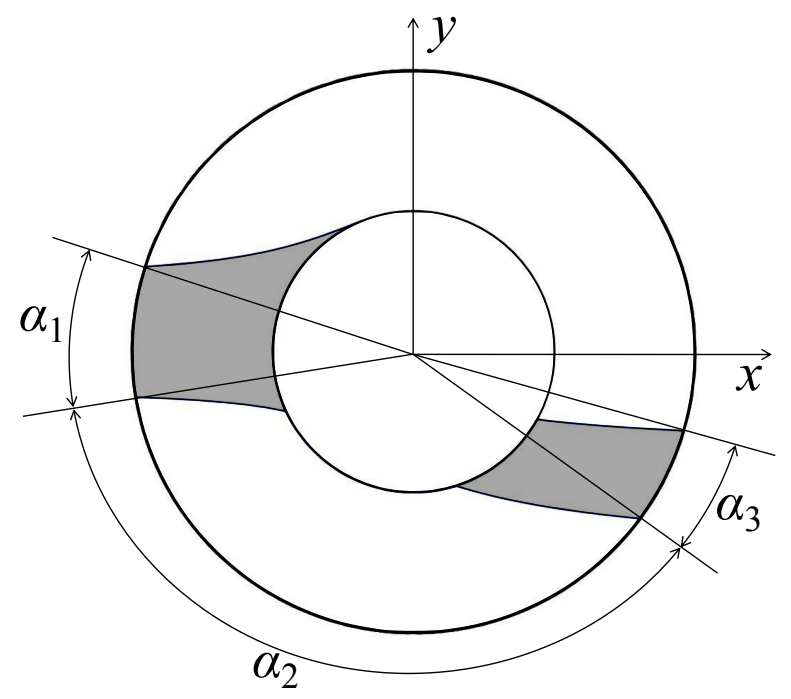

Figure 4 Representation of the angles necessary for the leakage computation

to $\alpha_{2}$. The power loss due to leakage between the trough and the blades is equal to

$$
P_{l}=\rho g Q_{l} H
$$

Leakage due to over-filling

The leakage due to the flow over the screw core appears when the flow rate is too high or the rotational speed too low. In this case, a triangular spillway is formed. Nuernbergk and Rorres (2012) suggest using the over-filling leakage model given by Aigner (2008) to estimate the flow rate

$$
Q_{\text {over }}=\frac{4}{15} \mu_{\text {over }} \sqrt{2 g}\left(\frac{1}{\tan \beta}+\tan \beta\right) h_{e}^{5 / 2}
$$

The weir coefficient $\mu_{\text {over }}$ shall be taken as $\mu=0.537$ (for maximum spillway leakage). The term $h_{e}$ corresponds to the height of the triangular spillway (Fig. 3). The power loss due to over-filling is then given by

$$
P_{\text {over }}=\rho g Q_{\text {over }} H
$$

\section{Friction of water on trough}

Friction force on trough is due to the viscous fluid motion through the ASG system. This force is providing work, then energy is dissipated. It is given by

$$
F_{\text {trough }}=\tau_{\text {trough }} A_{\text {trough }}=\frac{\lambda_{\text {trough }}}{8} \rho c_{\text {ax }}^{2} A_{\text {trough }}
$$

where $\tau_{\text {trough }}$ is the shear stress of fluid on trough and $A_{\text {trough }}$ is the wetted area of the trough,the latter being numerically determined. Assuming that we have a free-surface flow in a smooth channel, the shear stress is described with the friction law of Darcy-Weisbach where $\lambda_{\text {trough }}$ is the friction coefficient of water on the trough, $\rho$ is the fluid density and $c_{a x}$ is the average axial flow velocity 
in the trough. Assuming that this velocity is equal to the axial velocity of the blades, we have

$$
c_{a x}=\frac{S n}{60}
$$

Assuming that we have a semi-circular free-surface flow, the friction coefficient $\lambda_{\text {trough }}$ can be determined using the Manning equation for the axial velocity. The details of the calculations are exposed in Nuernbergk (2012) and are used without modifications. After determining $\lambda_{\text {trough }}$ and calculating $A_{\text {trough }}$, it is possible to compute $F_{\text {trough }}$. The work of the friction forces along the trough is then equal to

$$
\begin{aligned}
W_{\text {trough }} & =F_{\text {trough }} L_{B} \\
& =\rho V_{\text {water }} g h_{\text {trough }}
\end{aligned}
$$

where $V_{\text {water }}$ is the total volume of water and $h_{\text {trough }}$ is the head due to the friction. Then we have

$$
h_{\text {trough }}=\frac{F_{\text {trough }} L_{B}}{\rho V_{\text {water }} g}
$$

Finally, the power loss due to the friction on the trough is equal to

$$
P_{\text {trough }}=\rho g Q h_{\text {trough }}
$$

\section{Friction of water on screw core}

The friction of the fluid on the screw core generates a friction force due to fluid viscosity and then creates a resistant torque. This force is determined by

$$
F_{\text {core }}=\tau_{\text {core }} A_{\text {core }}
$$

where $\tau_{\text {core }}$ is the shear stress of fluid on screw core and $A_{\text {core }}$ is the wetted area of the screw core which is numerically determined. Again, for a free surface flow on a smooth surface, the friction is described with the Darcy-Weisbach's law and a constant friction coefficient. Then the shear stress $\tau_{\text {core }}$ is defined by

$$
\tau_{\text {core }}=\frac{\lambda_{\text {screw }}}{8} \rho c_{f / \text { core }}^{2}
$$

where $\lambda_{\text {screw }}$ is the friction coefficient of the fluid on the screw and $c_{f / c o r e}$ is the assumed fluid velocity relative to the core. This coefficient $\lambda_{\text {screw }}$ is experimentally determined. The fluid velocity $c_{f / c o r e}$ relative to the screw core is equal to $u_{r}$ where $u_{r}=2 \pi R_{i} n / 60$ is the radial component of the velocity. The brake torque due to this friction is then equal to

$$
C_{\text {core }}=F_{\text {core }} R_{i}
$$

Finally, the power loss due to the friction of the fluid on the screw core is given by

$$
P_{\text {core }}=C_{\text {core }} \omega
$$

where $\omega=2 \pi n / 60$ is the rotational speed of the screw. 


\section{Friction of water on blades surface}

As previously, fluid flow on the upper and lower wetted surfaces of blades generates a friction force due to fluid viscosity and then creates a brake torque. The elementary friction force is equal to

$$
\mathrm{d} F_{\text {blade }}=\tau_{\text {blade }} \mathrm{d} A
$$

where $\tau_{\text {blade }}$ is the shear stress of fluid on blades and $\mathrm{d} A=\mathrm{d} b \mathrm{~d} r$ is the elementary surface. The Darcy-Weisbach's law is used again to describe the friction. Then, the shear stress $\tau_{\text {blade }}$ is given by

$$
\tau_{\text {blade }}=\frac{\lambda_{\text {screw }}}{8} \rho c_{f / \text { blade }}^{2}
$$

where $c_{f / b l a d e}$ is the assumed fluid velocity relative to a blade and $\lambda_{\text {screw }}$ is the same friction coefficient than previously used for the friction on screw core. The elementary brake torque projected on the screw axis is equal to

$$
\mathrm{d} C_{f, \text { blade }}=\mathrm{d} F_{\text {blade }} r \cos \left(\beta_{e}\right)=\frac{\lambda_{\text {screw }}}{8} \rho\left(\frac{2 \pi n}{60}\right)^{2} r^{3} \cos \left(\beta_{e}\right) \mathrm{d} b \mathrm{~d} r
$$

The total brake torque is then equal to

$$
C_{f, \text { blade }}=\frac{\lambda_{R}}{8} \rho\left(\frac{2 \pi n}{60}\right)^{2}\left[\iint_{p, \text { sup }} \cos \left(\beta_{e}\right) r^{3} \mathrm{~d} b \mathrm{~d} r+\iint_{p, \text { inf }} \cos \left(\beta_{e}\right) r^{3} \mathrm{~d} b \mathrm{~d} r\right]
$$

The bounds of the integrals correspond to to the upper and lower wetted surface of a blade. The integrals are numerically approximated. The brake torque for all the screw length is equal to

$$
C_{f, \text { screw }}=\frac{L_{B} N}{S} C_{f, \text { blade }}
$$

Finally, the power loss due to fluid friction on the blades is determined by

$$
P_{\text {blade }}=C_{f, \text { screw }} \omega
$$

\section{Experimental device}

In order to investigate experimentally the ASG performances, an experimental device with a laboratory-scale Archimedean screw was elaborated. It is installed in the fluid mechanics laboratory at the INSA of Strasbourg. This device enables testing of the screw for different geometrical parameters and flow conditions. The experimental results will be compared to the theoretical ones obtained from the model exposed above. The flowrate, the inclination of the screw, the rotational speed and the downstream water level can be changed with the experimental device (Fig. 5). All geometrical parameters and flow conditions are represented in Table 1.

The experimental device is installed in a $0.75 \mathrm{~m}$ width and $5 \mathrm{~m}$ length open channel. The inlet and outlet have circular cross-sections of $0.15 \mathrm{~m}$ diameter. The Archimedean screw is manufactured in ABS with a 3D printer. The turbine is supported by two sealed ball bearings. The screw is directly coupled to the axis of a RE050G Maxon $(48 \mathrm{~V}, 418 \mathrm{~W}) \mathrm{DC}$ motor with steel bellows coupling. The rotational speed of the screw can be controlled by using the motor as a brake. Moreover, it is also used as a measurement device. Indeed, the rotational speed of the screw is proportional to the 
Table 1 Geometrical parameters and flow conditions

\begin{tabular}{clc}
\hline & Outer radius of the screw $-R_{a}(\mathrm{~m})$ & 0.096 \\
& Inner radius of the screw $-R_{i}(\mathrm{~m})$ & 0.052 \\
Geometrical parameters & Pitch of the screw $-S(\mathrm{~m})$ & 0.192 \\
& Threaded length of the screw $-L_{B}(\mathrm{~m})$ & 0.4 \\
& Number of blades $-N$ & 3 \\
& Inclination of the screw $-\beta\left(^{\circ}\right)$ & $18 \ldots 30$ \\
& Gap width between screw and trough $-s_{\text {sp }}(\mathrm{m})$ & 0.0007 \\
\hline \multirow{2}{*}{ Flow conditions } & Rotational speed of the screw $-n\left(\mathrm{~min}^{-1}\right)$ & $60 \ldots 180$ \\
& Flow rate $-Q\left(\mathrm{~m}^{3} \mathrm{~s}^{-1}\right)$ & $0.001 \ldots 0.004$ \\
& Water level downstream $-h_{\text {out }}(\mathrm{m})$ & $0 \ldots 0.25$ \\
& Water level upstream $-h_{\text {in }}(\mathrm{m})$ & $0.26 \ldots 0.35$ \\
\hline
\end{tabular}

voltage and torque to electrical current. Then we have the torque delivered by the motor defined by $C_{\text {motor }}=k_{\text {torque }} I$ and the rotational speed by $n=k_{\text {speed }} U$ where $k_{\text {torque }}$ is the torque constant, $I$ is the electric current at the motor terminals, $k_{\text {speed }}$ is the constant of speed and $U$ is the voltage at the motor terminals. It is important to note that $k_{\text {torque }}$ and $k_{\text {speed }}$ are independent of the motor efficiency in our range of rotational speed. In order to determine the value of the gap between trough and blades $s_{s p}$, we measured this gap with slip gauges at both ends of the screw for different radial positions. The maximal gap corresponding to $s_{s p}=0.0007$ is used. Water is supplied at the inlet of the experimental device with a centrifugal pump (Fig. 5). The fluid flows through the screw and leads to its rotation thanks to the pressure exerted on its blades. Finally, the water escapes at the outlet located downstream of the adjustable weir (Fig. 5). The downstream level is controlled with an adjustable weir. The flowrate is measured with a PROMAG 30F Endress Hauser magnetic flowmeter with a given measuring accuracy of $\pm 0.5 \%$. The downstream and upstream water levels are measured with dial gauges. For each operating point, corresponding to one flow condition and one rotational speed, 1500 instantaneous values of rotational speed and torque are recorded in 15 s. These measures give an average rotational speed and torque. Subsequently, these average values are used for analyzing the performances of the screw. The measures are recorded and analyzed with the software Labview 2013 through a National Instrument data acquisition device (NI USB $6008)$.

The power produced by the screw is determined by

$$
P_{\text {screw }}=C_{\text {screw }} \omega
$$

where $P_{\text {screw }}$ is the power provided by the screw and $C_{\text {screw }}$ is the torque provided by the screw. The torque provided by the screw is equal to

$$
C_{\text {screw }}=C_{\text {motor }}+C_{\text {friction }}
$$

where $C_{\text {motor }}$ is the brake torque delivered by the motor and $C_{\text {friction }}$ is the torque induced by the friction in the bearings. In order to know the real screw torque, the friction torque $C_{\text {friction }}$ was estimated by measuring the motor torque necessary for rotating the screw for different rotational speeds. It appears that this torque increases linearly with the speed and a linear regression gives

$$
C_{\text {friction }}(n)=0.000171 n+0.046065
$$

As we use preloaded bearings, we expect that the equation 34 does not change significantly with a screw partially or completely filled. The turbine efficiency is then equal to

$$
\eta=\frac{P_{\text {screw }}}{P_{\text {hydraulic }}}=\frac{C_{\text {screw }} \omega}{\rho g Q H}
$$




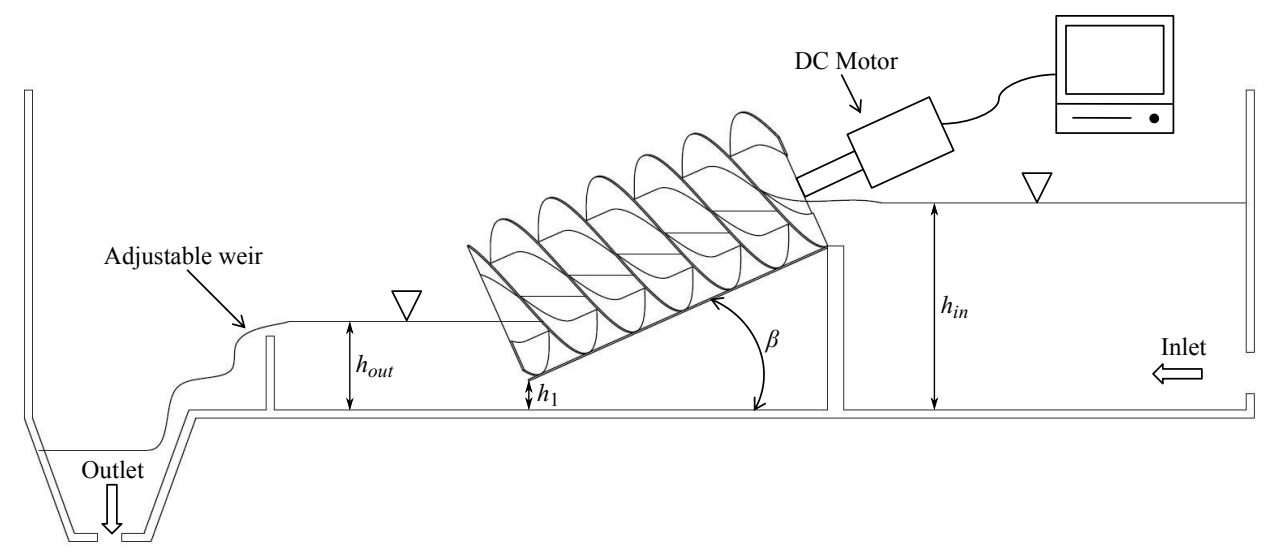

Figure 5 Experimental device

where $P_{\text {hydraulic }}$ is the hydraulic power provided by the moving fluid.

\section{Results and Discussion}

\subsection{Impact of downstream level on $A S G$ performances}

According to Lyons and Lubitz (2013), the downstream water level has a direct impact on ASG performances. In the present study, experimental measurements are performed in order to determine optimal operating points for various downstream water levels and screw inclinations. These results could be useful for ASG design and site selection in real river networks. The screw performances are tested for different outlet levels $h_{\text {out }}$ and inclinations at fixed speed and flow rate. Downstream water level is controlled with an adjustable weir (Fig. 5).

To compare the results, we define the screw immersion that corresponds to the following ratio

$$
I=\frac{h_{\text {out }}-h_{1}}{2 R_{a} \cos (\beta)}
$$

where $h_{1}$ is represented in Fig. 5. Thus, for $I=0$, the water level is at the low end of the screw . Conversely, for $I=1$, the end of the screw is completely immersed. Different levels of immersion are represented in Fig. 6. According to Nuernbergk (2012), the optimal downstream level $h_{\text {out,opti }}$ is obtained from

$$
h_{\text {out }, \text { opti }}=\left(R_{a}+R_{i}\right) \sqrt{1-\left(\frac{\tan (\beta) S}{2 \pi R_{i}}\right)^{2}} \cos (\beta)-\frac{S}{N} \sin (\beta)+h_{1}
$$

The rotational speed is fixed at $n=80 \mathrm{~min}^{-1}$ and the flowrate at $Q=3 \mathrm{ls}^{-1}$ for all these tests.

Figure 7 shows the experimental efficiency and the torque depending on the immersion $I$ for a screw inclination of $24^{\circ}$. It also shows the theoretical optimal immersion $I_{\text {opt }}$ obtained from the equations (36) and (37). Note that the torque provided by the screw decreases when $I$ is decreasing. This can be explained by the fact that downstream water exerts pressure on the last blade of the screw. Thus, this pressure that brakes the screw increases when the water level is increasing and then, reduces the torque. It can be observed on Fig. 7 that given $(\beta, n, Q)$ the efficiency increases with $I$ before reaching a maximum for $I$ around 0.64 . Once this value is reached, the efficiency decreases while the immersion continues to increase. Thus, there is only one optimal value of $I$. 


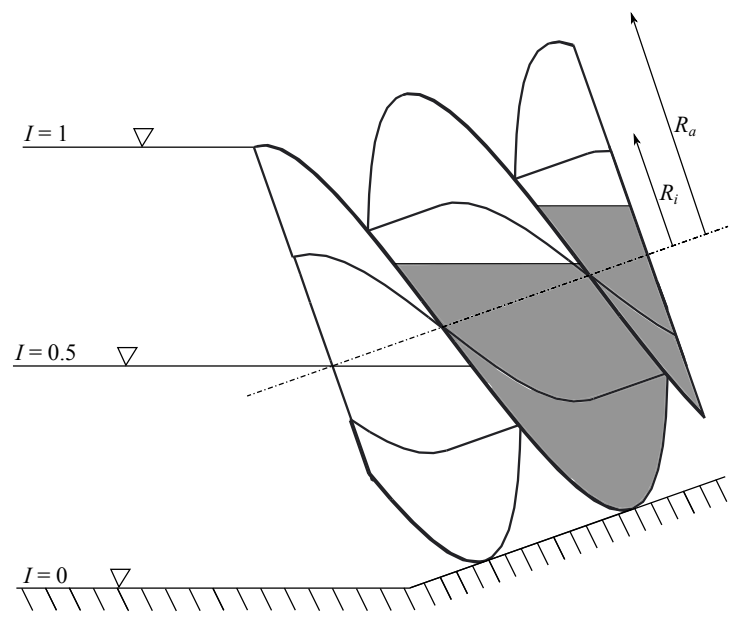

Figure 6 Representation of outlet level for $I=0, I=0.5$ and $I=1$

These results show that up to $20 \%$ of efficiency can be lost with a bad value of $I$. This sensitivity to imersion $I$ might be increased due to the laboratory-scale of the screw. However, this significant impact of the screw immersion on efficiency is in good agreement with the results found in Lyons and Lubitz (2013). In order to obtain the best screw performances it is therefore necessary to reach this level of immersion. It may be noted that we obtain the same evolution of the efficiency and torque as a function of $I$ for both $\beta=20^{\circ}$ and $\beta=28^{\circ}$.

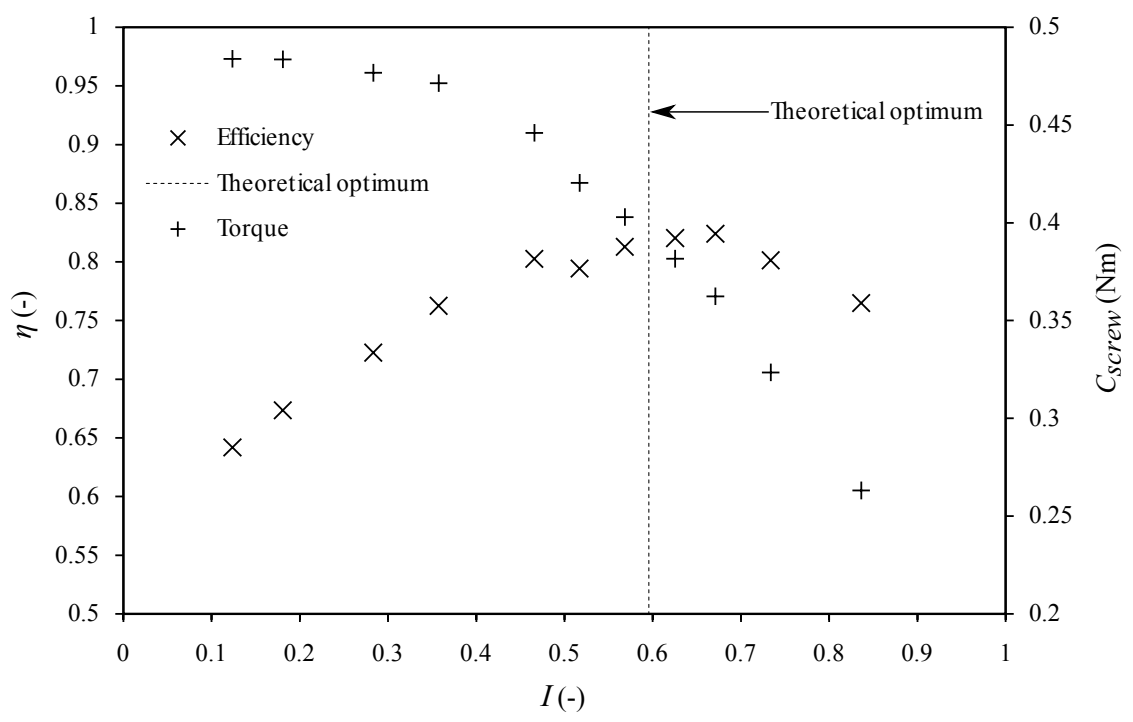

Figure 7 Experimental efficiency $\eta$ and torque $C_{\text {screw }}$ depending on the screw immersion $I$ with the theoretical optimum immersion for $\beta=24^{\circ}, n=80 \mathrm{~min}^{-1}$ and $Q=3 \mathrm{ls}^{-1}$

The optimal downstream level corresponds to the water level in the last bucket minus the height $\delta h=S \sin (\beta) / N$. When the downstream level is above this line, the water can flow back into the screw. This phenomenon brakes the screw and then reduces its performances. When the outlet level is $x$ meter under the optimal line for example, the torque provided by the screw will increase because of the diminution of the hydrostatic pressure on the last blade. In return, the hydraulic 
power delivered by the flow will increase of $\rho g Q x$. Unfortunately, the gain in hydraulic power is superior to the gain in mechanical power and as we have $\eta=P_{\text {screw }} / P_{\text {hydraulic }}$, the efficiency will eventually decrease.

\begin{tabular}{|c|c|c|}
\hline & \multicolumn{2}{|c|}{ Optimal immersion - $I_{o p t}$} \\
\hline Inclination $\left({ }^{\circ}\right)$ & Theoretical & Experimental \\
\hline 20 & 0.632 & 0.67 \\
\hline 24 & 0.596 & 0.64 \\
\hline 28 & 0.536 & 0.57 \\
\hline
\end{tabular}

Table 2 exposes the theoretical and experimental optimums of immersion for different screw inclinations - figures not presented. Remark that experimental values are slightly higher than the theoretical values but show the same trend: both theoretical and experimental values of $I_{o p t}$ decrease when the screw inclination increases. This evolution can be explained by the geometry. Finally, the equation (37) gives a good estimation of the optimal downstream water level. We also noticed that the downstream water level impacts directly the screw sound emission. Indeed it was noted qualitatively that the sound emission is louder for lower immersion levels. In the next part, all the experimental results are obtained with a screw operating at optimal immersion.

\subsection{Comparison to theoretical model}

In order to evaluate the theoretical model described in the previous part, theoretical results are compared to experimental measurements. Two measurement sets of screw efficiency and axial torque are performed. A firstset is done with variable flowrates and fixed rotational speed while a second set is done with constant flowrate and variable rotational speeds. For each set, the screw is tested for three different inclinations. Geometrical parameters and flow conditions for these experiments are exposed in Table 3.

Table 3 Flow conditions and geometrical parameters for experiments at variable rotational speed and variable flow rate

\begin{tabular}{ccccccc}
\hline & \multicolumn{3}{c}{ Variable speed } & \multicolumn{3}{c}{ Variable flow rate } \\
\hline Inclination $\left(^{\circ}\right)$ & $Q\left(\mathrm{ls}^{-1}\right)$ & $I$ & $n_{n o m}\left(\min ^{-1}\right)$ & $n\left(\min ^{-1}\right)$ & $I$ & $Q_{n o m}\left(\mathrm{ls}^{-1}\right)$ \\
20 & 3 & 0.64 & 84.2 & 90 & $0.60 \ldots 0.65$ & 3.2 \\
24 & 3 & 0.60 & 90.6 & 90 & $0.57 \ldots 0.61$ & 2.98 \\
28 & 3 & 0.54 & 98.7 & 90 & $0.5 \ldots 0.55$ & 2.73 \\
\hline
\end{tabular}

The results obtained with the theoretical model are highly dependent on the total head $H$. Knowing the weir height and the flow rate, it is possible to determine the evolution of the downstream water level. But it gets more difficult to determine the upstream water level. Although Nuernbergk and Rorres (2012) developed a model to predict water inflow of an Archimedean screw, the results obtained with this model do not corroborate our measurements. It is probably due to the size of the laboratory-scale screw. To avoid this difficulty, the experimental values of $H$ are used in our efficiency model.

To obtain the theoretical torque $C_{t h}$, the brake torques due to friction are subtracted from the torque $C_{t h, s c r e w}$. So, we have

$$
C_{t h}=C_{\text {th,screw }}-C_{f, \text { screw }}-C_{\text {core }}
$$

The torques $C_{t h, \text { screw }}, C_{\text {core }}$ and $C_{f, \text { screw }}$ derive respectively from the equations 9,24 and 30 . The 
value of the coefficient $\lambda_{\text {screw }}$ is adjusted until the theoretical and experimental efficiencies match. The same value of $\lambda_{\text {screw }}$, approximatively 0.084 , is found for variable flowrates or rotational speeds, and for each inclination. Nuernbergk (2012) found a value of this coefficient equal to 0.035 based on the experimental results of Brada (1999). The fact that our friction coefficient is much higher can be explained by the larger roughness of our screw. The theoretical values of efficiency and axial torque presented below derived respectively from the equations 11 and 38 .

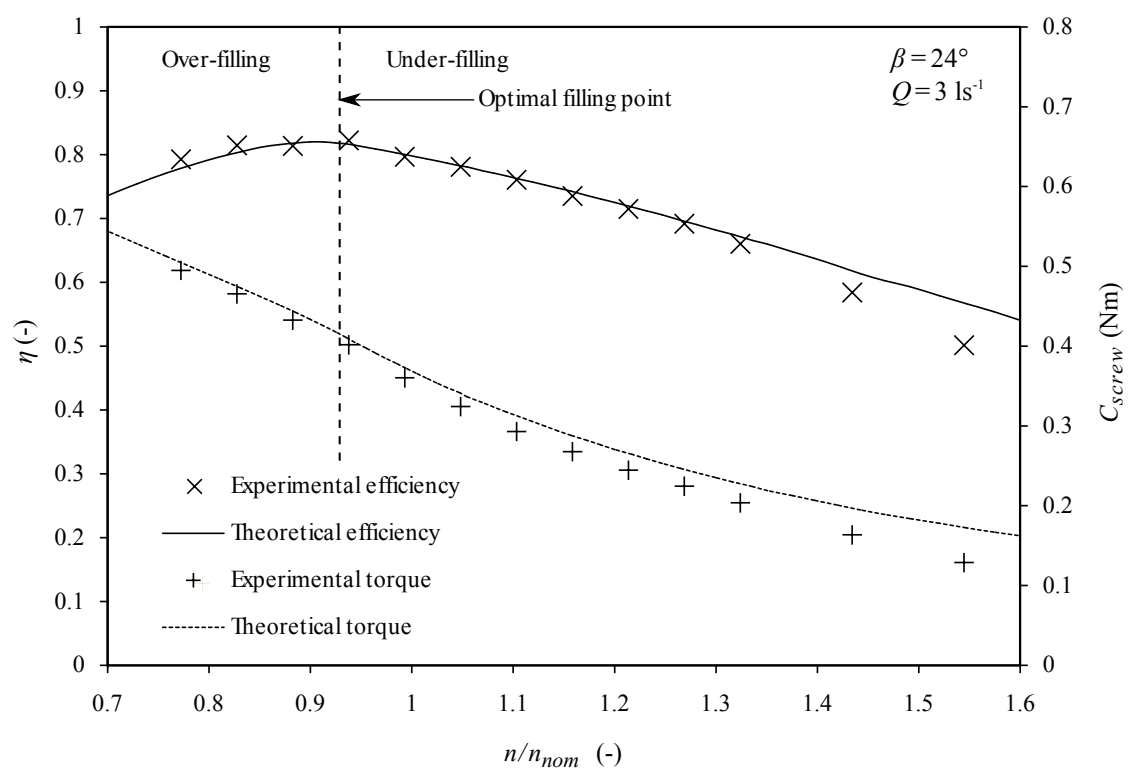

Figure 8 Experimental and theoretical (from equations 11 and 38) values for efficiency and axial torque depending on $n / n_{n o m}$ with $\beta=24^{\circ}$ and $Q=3 \mathrm{ls}^{-1}$

Figure 8 exposes the evolution of the screw efficiency and axial torque depending on $n / n_{\text {nom }}$ for $\beta=24^{\circ}$ and $Q=3 \mathrm{ls}^{-1}$. We can see that with the increase of the rotational speed, the experimental efficiency increases until reaching a maximum value. For higher values of rotational speed the efficiency decreases. There is only one optimal speed which corresponds here to $n / n_{\text {nom }}=0,95$. The maximum efficiency is slightly above $80 \%$, this is in good agreement with the laboratory results found in the literature (80\% in Lubitz et al. (2014), $80 \%$ in Brada (1999) and 90\% in Lashofer et al. (2013)). We note that the best efficiency is obtained for a slight over-filling for each screw inclination. The performance degradation with low speed is mainly due to over-filling which leads to leakage over the screw core. Conversely, when the speed is too high, the screw is under-filled. Gap leakage and friction forces will be predominant. Figure 8 shows that the torque decreases for increasing rotational speed. It is due to the screw filling that decreases when $n$ increases at constant flowrate. The pressure on the blades, as well as the torque, is then decreasing. Moreover, when the rotational speed increases, the friction forces that brake the screw increase too. For low speed, we notice a good agreement between theoretical and experimental values of efficiency and torque. It is particularly interesting to see that the theoretical model gives the same optimal value of $n / n_{\text {nom }}$. It shows the importance of taking into account the friction losses in the model and it can partially explain why Lubitz et al. (2014) obtained theoretical efficiencies much greater than the experimental ones. It should be noted that the theoretical torque is slightly higher than measurements with the friction coefficient $\lambda_{\text {screw }}$ defined previously. This difference can be explained by the fact that the term $L_{b} N / S$ in the equation (9) may lead to an overestimation of the torque $C_{\text {screw }}$. When the ratio $n / n_{\text {nom }}$ exceeds 1.3 , we can see that the experimental efficiency decreases. The same phenomenon occurs for $\beta=20^{\circ}$ and $\beta=28^{\circ}$ for the same value of $n / n_{\text {nom }}$ 
and therefore for the same filling point. We assume that gap leakage is underestimated above this point. Indeed, leakage tests were performed for the screw standing at the optimal and at $60 \%$ filling points. We found good agreement between theoretical and experimental leakage which are comparable when the screw is totally filled. However for low filling, we obtained respectively a theoretical and experimental leakage decrease of about $25 \%$ and $15 \%$. At low filling, the theoretical model hence predicts less leakage than in reality. This may explain as well the experimental torque diminution compared to the theory at high rotational speed.

Figure 9 shows the different ratios of power loss to hydraulic power depending on $n / n_{\text {nom }}$. We can see first that the friction losses increase quickly with the rotational speed, especially for the friction on blades that exceeds $30 \%$ for high rotational speed. The gap leakage decreases slowly when the speed increases but is still close to $5 \%$. When the screw is over-filled, we see that the over-filling leakage increases quickly to reach $10 \%$ and becomes the most important source of loss for $n / n_{\text {nom }}=0.7$. At optimal filling point, the two most important losses are the friction of water on the blades surface and gap leakage. Thus, the screw efficiency is strongly depending on the friction forces and not only on gap leakage. However, the friction forces are very important here because of the roughness of the screw.

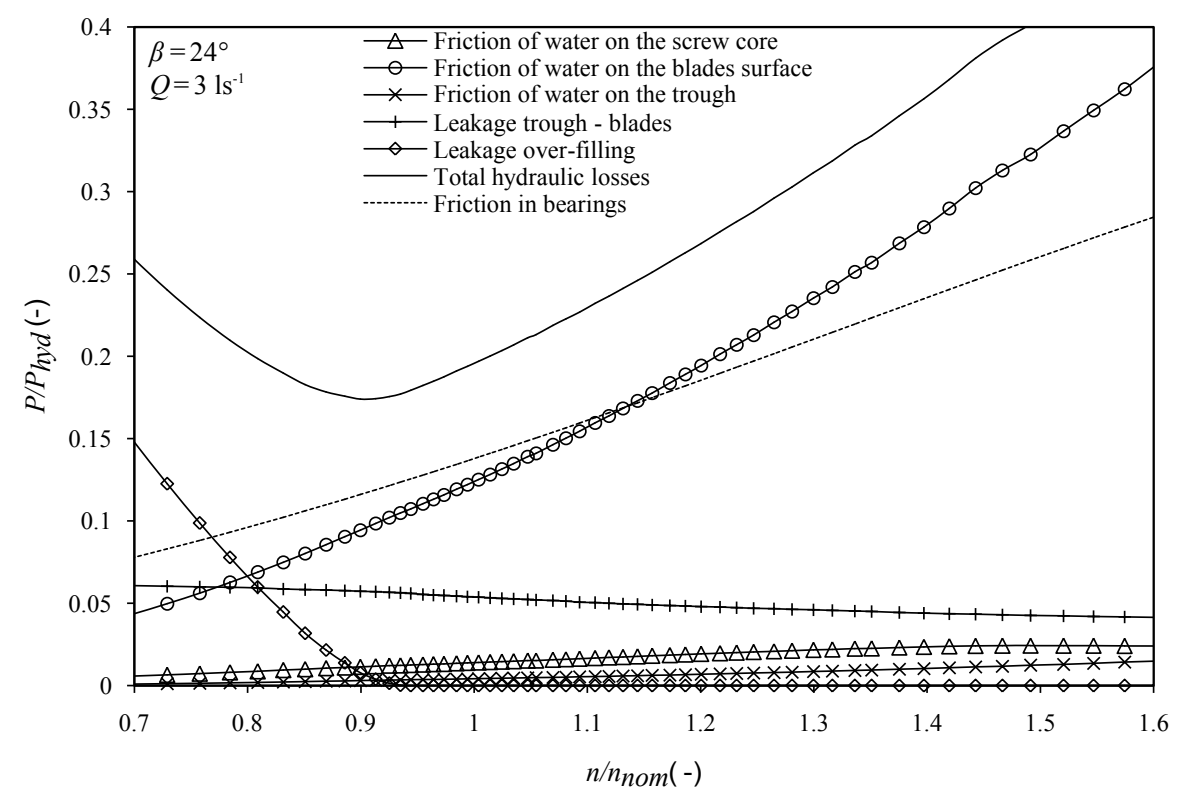

Figure 9 Evolution of different theoretical losses plus experimental bearings losses depending on $n / n_{n o m}$ with $\beta=24^{\circ}$ and $Q=3 \mathrm{ls}^{-1}$

Figure 10 exposes the evolution of the screw efficiency and axial torque depending on $Q / Q_{\text {nom }}$ for $\beta=24^{\circ}$ and $n=90 \mathrm{~min}^{-1}$. As previously, we can see that there is only one optimal flowrate that gives the highest efficiency. This optimal corresponds to $Q / Q_{\text {nom }}=1.08$. We obtain the same value for $\beta=20^{\circ}$ and $\beta=28^{\circ}$. Moreover, for each inclination we obtain the highest efficiency when the screw is slightly over-filled. As before, the efficiency decreases when the screw is in overfilling situation because of the leakage over the screw core. In under-filling situation, we note that the efficiency is only very slightly affected until $Q / Q_{\text {nom }}=0.8$. Below this value, the efficiency decreases quickly for each screw inclination. Thus, efficiency is affected only above a given filling point. We assume that this is due to the leakage between trough and blades which becomes more important from this point. Thus, there is only a small decrease in ASG efficiency for a $\pm 20 \%$ variation of discharge around the optimal value. This is in good agreement with the experimental results of Brada (1993) and Lashofer et al. (2013). For ASG with variable speed, good efficiencies can be maintained over a wider range of operating conditions. However, according to Lashofer et al. 
(2012), this kind of ASG is much more expensive and such a device is only relevant with significant river flow fluctuations. As can be expected, the torque decreases when the flow rate is decreasing.

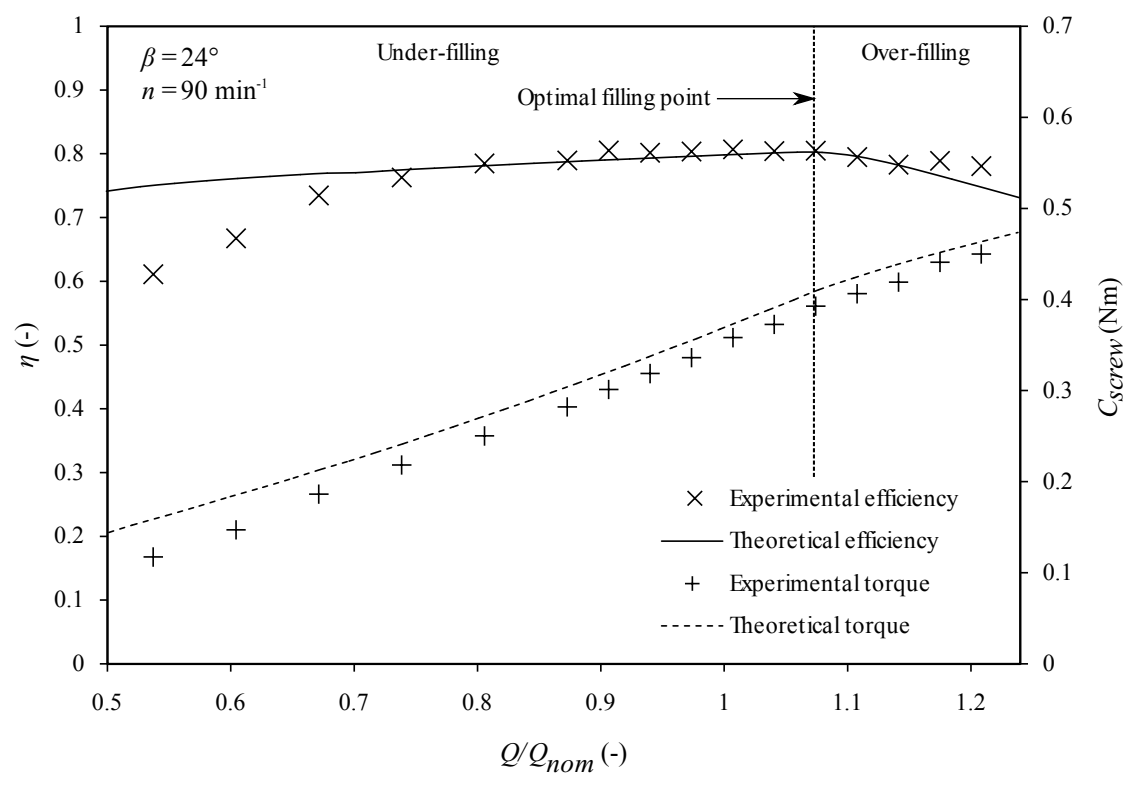

Figure 10 Experimental and theoretical (from equations 11 and 38) values for efficiency and axial torque depending on $Q / Q_{\text {nom }}$ with $\beta=24^{\circ}$ and $n=90 \mathrm{~min}^{-1}$

It can be explained, as previously, by the diminution of pressure on the blades when the screw filling is decreasing. Once again we see that there is a good agreement between experimental and theoretical values of efficiency and torque. Moreover, theory and experiments give the same optimal value of $Q / Q_{\text {nom }}$. But below $Q / Q_{\text {nom }}=0.8$, the experimental values decrease more quickly than theoretical ones. The axial torque values obtained by the theoretical model are slightly smaller than those measured experimentally, with the friction coefficient defined previously. This difference is more pronounced for low flow values. As previously, we think that the differences in efficiency and torque for low flow values stem from the underestimation of the gap leakage.

Figure 11 gives the different ratios of power loss to hydraulic power depending on $Q / Q_{n o m}$. We see that the two most significant losses are the friction on blades and the gap leakage loss and that their maximum value is respectively of $15 \%$ and $7 \%$. Both are decreasing slowly when the flow rate increases. As expected, the loss due to over-filling leakage increases quickly as soon as we are above the optimal filling point. Once again, these results demonstrate the importance of taking into account the friction forces. To obtain better performances, the value of $\lambda_{\text {screw }}$ and $s_{s p}$ must be minimized.

\section{Conclusion}

Allthough Archimedian screw used as turbine demonstrate a good potential for hydropower generation, low-head sites are still under-exploited and guidelines are still missing for an improved design of ASGs. This paper presents an improved theoretical model that predicts the performances of an Archimedean screw generator. It is based on the analysis and modelling of several physical phenomena. The first part of the model computes the axial torque provided by the screw from the pressure exerted by the fluid on the blades. The second part gives the screw efficiency from empirical equations for variable filling points. Subsequently, our theory is confronted to experimental measurements thanks to a laboratory-scale Archimedean screw. This device enables the 


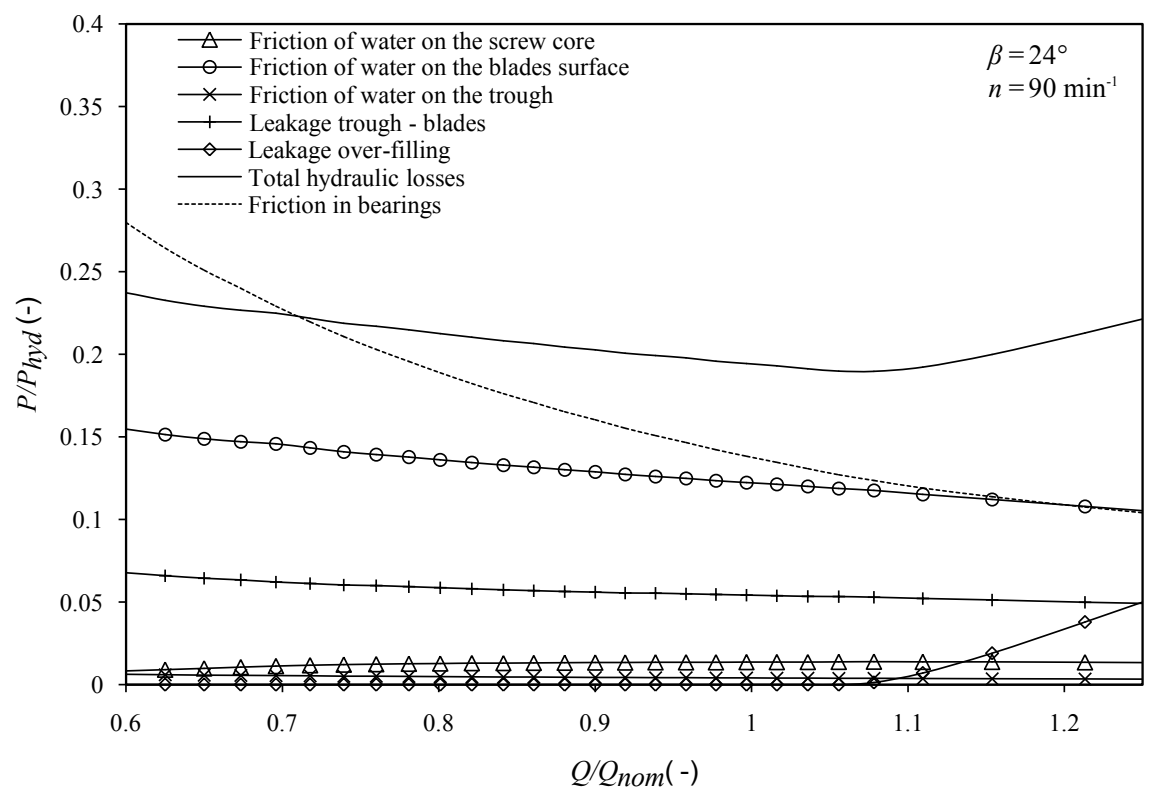

Figure 11 Evolution of different theoretical losses plus experimental bearings losses depending on $Q / Q_{\text {nom }}$ with $\beta=24^{\circ}$ and $n=90 \mathrm{~min}^{-1}$

investigation of the hydraulic performances of an ASG.

Experiments show the critical influence of downstream water level on the screw performances. Moreover the existence of an optimal point of screw immersion depending on the inclination is highlighted. By comparing with theoretical outlet level given by Nuernbergk (2012), we find experimental values slightly higher for each inclination.

To evaluate the theoretical model, two campaigns of measurements have been done with, in each case, three different screw inclinations. The first campaign was performed at a constant flowrate and a variable rotational speed and, the second one, at a fixed rotational speed and a variable flowrate. In each case a good agreement between experimental and theoretical values of efficiency and torque is found above a certain point of filling. Thus, for low filling, experimental values of torque and efficiency decrease quicker than the theoretical values. We assume that this phenomenon is due to underestimation of leakage gap at low filling. This study highlighted the importance of taking into account the friction forces in an ASG. Thus, in order to optimize the screw performances, it is essential to minimize the gap between the blades and trough but also to minimize the value of the friction coefficient between the screw and the fluid.

Finally, the theoretical model presented can be used to predict the ASG performances and especially to determine optimal flow rate or rotational speed. To be effective at low screw filling, the model of gap leakage will be improved. More experiments will be done for new screw geometries and friction coefficients. In addition, the model used will be confronted to a real-scale Archimedean screw.

\section{Acknowledgements}

The authors gratefully aknowledge all the partners involved in the project MCH funded by FUI (Fonds Unique Interministriel). We also want to thank the three reviewers that helped to significantly improve the paper. 


\section{Notation}

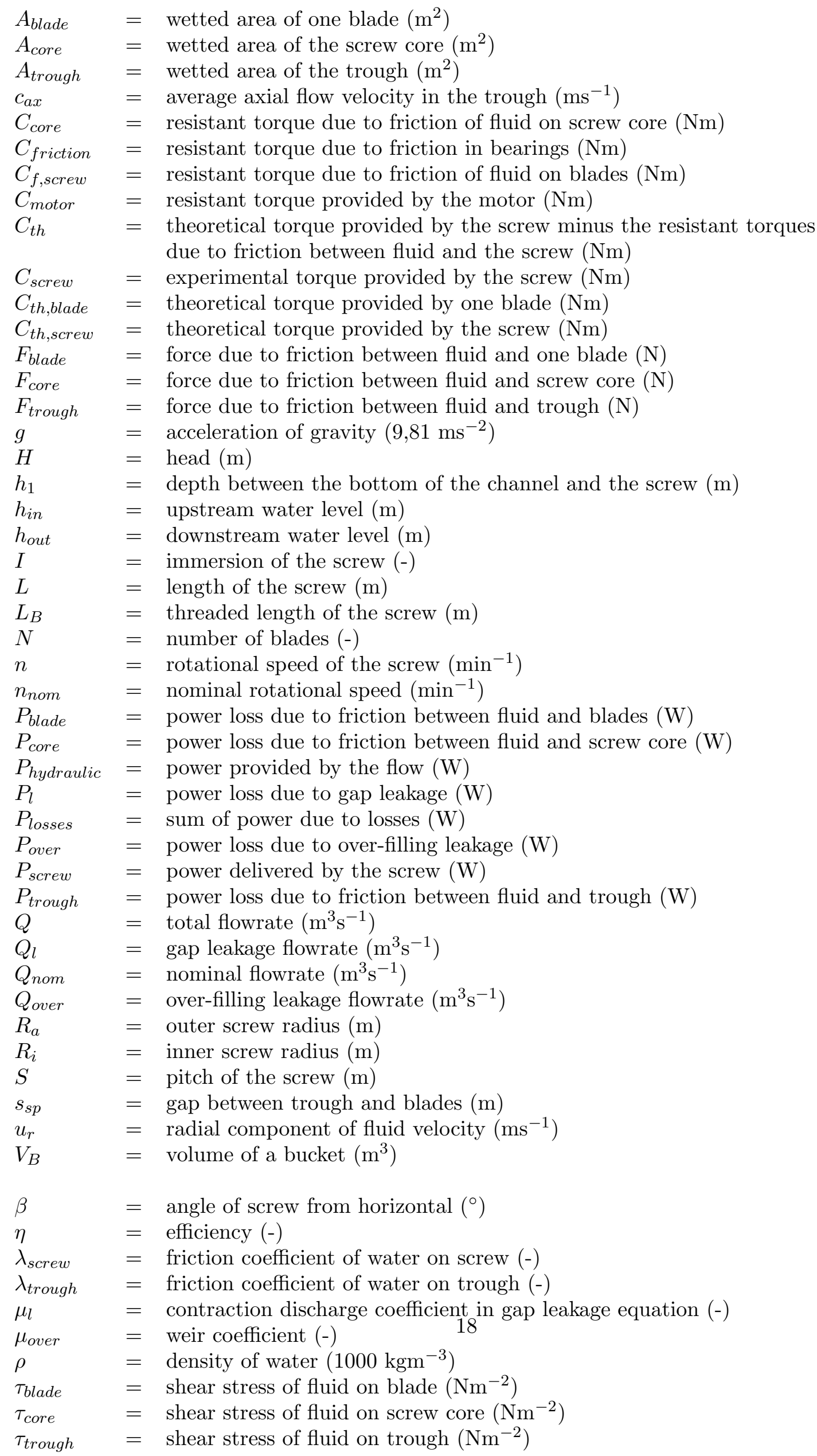




\section{References}

Aigner, D. (2008). Aktuelle forschungen in wasserbau 1993-2008. In S. der Technischen Universität Dresden (Ed.), (Vol. 36, p. 162-176). Dresden, Germany: Institut für Wasserbau und Technisch Hydromechanik de TU Dresden.

Brada, K. (1993). Einaches kleinkraftwerke mit schneckenturbine zur betrieblichen stromerzeugung. MM. Maschinenmarkt, 99, 30-32.

Brada, K. (1999). Wasserkraftschnecke ermöglicht stromerzeugung über kleinkraftwerke. MM. Maschinenmarkt, 105, 52-56.

Kiebel, P. (2007). Fish monitoring and live fish trials. phase 1 report: Livefish trials, smolts, leading edge assesment, disorientation study, outflow monitoring. (Tech. Rep.). Fishtek Consulting.

Kiebel, P. (2008). Archimedes screw turbine fisheries assessment. phase 2: Eels and kelts. (Tech. Rep.). Fishtek Consulting.

Kiebel, P., \& Coe, T. (2011). Archimedean screw risk assessment: strike and delay probabilities. (Tech. Rep.). Fishtek Consulting.

Lashofer, A., Hawle, W., \& Pelikan, B. (2012). State of technology and design guidelines for the archimedes screw turbine. In Hydro 2012. Wallington, Surrey, U.K..

Lashofer, A., Hawle, W., \& Pelikan, B. (2013). Betriebsbereiche und wirkungsgrade der wasserkraftschnecke. Wasserwirtschaft, 103, 29-34.

Lubitz, W. D., Lyons, M., \& Simmons, S. (2014). Performance model of archimedes screw hydro turbines with variable fill level. Journal of Hydraulic Engineering, 140(10), 04014050.

Lyons, M., \& Lubitz, W. D. (2013). Archimedes screws for microhydro power generation. In Asme 2013 7th international conference on energy sustainability. New York.

Müller, G., \& Senior, J. (2009). Simplified theory of archimedean screws. Journal of Hydraulic Research, 47(5), 666-669.

Muysken, J. (1932). Calculation of the effectiveness of the auger. De Ingenieur, 21, 77-91.

Nagel, G., \& Radlik, K.-A. (1988). Wassserforderschnecken. Bauverlag.

Nuernbergk, M. (2012). Wasserkraftschnecken: Berechnung und optimaler entwurf von archimedischen schnecken als wasserkraftmaschine. (Detmold, Ed.). Verlag Moritz Schäfer.

Nuernbergk, M., \& Rorres, C. (2012). Analytical model for water inflow of an archimedes screw used in hydropower generation. Journal of Hydraulic Engineering, 139(2), 213-220.

Radlik, K.-A. (1997). Wasserkraftschnecke zur energieumwandlung [hydrodynamic screw for energy conversion] de no. 4,139,134. Google Patents.

Rorres, C. (2000). The turn of the screw: Optimal design of an archimedes screw. Journal of Hydraulic Engineering, 126(1), 72-80.

Williamson, S., Stark, B., \& Booker, J. (2014). Low head pico hydro turbine selection using a multi-criteria analysis. Renewable Energy, 61, 43-50. 